\title{
Influence of inter-electron scattering on the form of non-equilibrium distribution function of band carriers
}

\section{I.I. Boiko}

V. Lashkaryov Institute of Semiconductor Physics, NAS of Ukraine

45, prospect Nauky, 03680 Kyiv, Ukraine,

E-mail: igoroksanaboiko@gmail.com

Phone (380-44)236-5422

\begin{abstract}
Consideration of different problems of physical kinetics shows that nonequilibrium distribution function of band carriers and, consequently, kinetic coefficients significantly depend on the specific shape of bands and mutual interaction of carriers. The most impressive results are related with interband scattering of mobile particles belonging to many-valley systems.
\end{abstract}

Keywords: kinetic equation, collision integral, non-equilibrium distribution function, many-valley system.

doi: https://doi.org/10.15407/spqeo21.02.114

PACS 72.10.-d, 72.20.-i

Manuscript received 19.03.18; revised version received 18.06.2018; accepted for publication 27.06.2018; published online 03.07.18.

\section{Introduction}

The main problem of physical kinetics is construction of a well-grounded kinetic equation and solution of it to obtain non-equilibrium distribution function (see, for example, [1-4]). The second step is to evaluate kinetic coefficients (see [5-9]). The first problem has no well satisfactory solution up to date. The main trouble is related with the specific form of kinetic equation (that is an integer-differential equation with specific derivatives). Today, there is no reliable way to find analytically precise solution of it, and in practice one has to construct and use some suitable approximations. One typical way is to neglect $e$-e-collisions of band charged carriers, but this way sometimes is not confident. Investigation shows that these collisions can be especially important for complex system of different types of band carriers (for example, system different of band-valleys).

2. One-particle density matrix for non-equilibrium many-particle system of charged carriers

Design by the symbols $A, B$ etc. some quantum numbers that characterize states of separate particles, which make up a system of charged band carriers. For uniform space, we assume the notation $A \rightarrow \vec{k}_{A}$, where $\vec{k}_{A}$ is the wavevector.

If the system of charged carriers is separated by several distinctive parts, we design these parts by the chosen symbols $p$ or $g$ that belong to the used set of numbers:

$(p$ or $g)=a, b, c$ etc.
Let the values $\Psi_{A}{ }^{p}(\vec{r})$ or $\Psi_{A}^{g}(\vec{r})$ are basic one-particle wave-functions. In what follows, the spin variables and spin quantum numbers are not applied, with account of processes of spin overturn they are not considered here.

The one-particle density matrix for $p$-carriers is defined in the following way:

$\rho_{A B}^{p p}(t) \equiv \rho_{A B}^{p}(t)=a_{B}^{+p}(t) a_{A}^{p}(t)$.

The cross-particle density matrix is as follows:

$\rho_{A B}^{p g}(t)=a_{B}^{+g}(t) a_{A}^{p}(t)$

Here, $t$ is time, $a_{A}^{+p}$ and $a_{A}^{p}$ are operators of generation and annihilation of band $p$-particles, the state of which is marked as $A$. The averaged value of density matrix (2.1):

$f_{A B}^{p}(t)=\left\langle\rho_{A B}^{p}(t)\right\rangle=\left\langle a_{B}^{+p}(t) a_{A}^{p}(t)\right\rangle$.

Averaging procedure \langle\rangle is presented by angle brackets; formally it is performed using the non-equilibrium statistical operator related to all the band carriers and to external scattering system together. The latter is presented by intrinsic accidental microscopic fields and macroscopic electrical field $\vec{E}$ (see [1-5]).

Now write the set of equations for one-particle density matrices $\rho_{A B}^{p}(t)$ relating to the chosen $p$ particles. As the start point, one uses the standard motion 
equations for operators $\rho_{A B}^{p}(t)$ at Heisenberg representation (see, for instance [3]):

$i \hbar \frac{\partial \rho_{A B}^{p}(t)}{\partial t}=\left[\rho_{A B}^{p}(t), \hat{H}_{p}^{t o t}\right] \equiv \rho_{A B}^{p}(t) \hat{H}_{p}^{t o t}-\hat{H}_{p}^{t o t} \rho_{A B}^{p}(t)$

Here, the total Hamiltonian $\hat{H}_{p}^{\text {tot }}$ of considered $p$-system is the sum of four parts:

$$
\hat{H}_{p}^{t o t}=\hat{H}_{p}+\hat{H}_{S}+\hat{H}_{p S}+\sum_{g} \hat{H}_{p g}
$$

Here, the Hamiltonian $\hat{H}_{p}$ concerns carriers interacting with macroscopic external fields, individual Hamiltonian $H_{S}$ relates to external scattering system of impurities and phonons (see, for instance [6]) and Hamiltonian $H_{p S}=e_{p} \varphi_{S}$ describes the interaction of $p$-carriers (farther we call them for simplicity as electrons) with external scattering system, Hamiltonian $\hat{H}_{p g}$ represents the mutual $p$ - $g$-interaction. The macroscopic electric field is directed along $z$-axis:

$\vec{E}=\left(0,0, E_{z}\right)$.

The first term in the right part of (2.5) is

$$
\begin{aligned}
& \hat{H}_{p}=\sum_{A B}\left(\hat{H}_{p}\right)_{A B} a_{A}^{+p} a_{B}^{p}= \\
& =\sum_{A B}\left[\left(\hat{H}_{p}^{(0)}\right)_{A B}+\left(\hat{H}_{p}^{(E)}\right)_{A B}\right] a_{A}^{+p} a_{B}^{p}= \\
& =\sum_{A B}\left[\varepsilon_{A B}^{p} \delta_{A B}-e_{p} E_{z}(z)_{A B}^{p}\right] a_{A}^{+p} a_{B}^{p} .
\end{aligned}
$$

Hamiltonian of Coulomb interaction of band carriers has the following form (see [10]):

$$
\hat{H}_{p g}=\sum_{A B A^{\prime} B^{\prime}} V_{A B A^{\prime} B^{\prime}}^{p g} a_{A}^{+p} a_{A^{\prime}}^{+g} a_{B^{\prime}}^{g} a_{B B}^{p}
$$

where

$$
V_{A B A^{\prime} B^{\prime}}^{p g}=-\frac{e^{2}}{2 \varepsilon_{L}} \int d^{3} \bar{r} \int d^{3} \vec{r}^{\prime} \Psi_{A}^{* p}(\vec{r}) \Psi_{A^{\prime}}^{* g}\left(\vec{r}^{\prime}\right) \frac{1}{\left|\vec{r}-\vec{r}^{\prime}\right|} \Psi_{B^{\prime}}^{g}\left(\vec{r}^{\prime}\right) \Psi_{B}^{p}(\vec{r}) .
$$

Hamiltonian $\hat{H}_{p S}$ has the form concerning the scattering potential:

$$
\hat{H}_{p S}=e_{p} \sum_{A B}\left(\hat{\varphi}^{(S)}\right)_{A B} a_{A}^{+p} a_{B}^{p}=e_{p} \sum_{A B}\left(\hat{\varphi}^{(S)}\right)_{A B} \rho_{B A}^{p} .
$$

Below, we omit the term that simply shows a shift of origin for counting out the kinetic energy. As a result, one obtains total Hamiltonian in the form

$$
\begin{aligned}
& \hat{H}_{p}^{\text {tot }}=\sum_{A B}\left\{\left(\hat{H}_{p}\right)_{A B}+e_{p}\left(\hat{\varphi}^{(S)}\right)_{A B}\right\} \hat{\rho}_{B A}^{p}+ \\
& +\sum_{g} \sum_{A B A^{\prime} B^{\prime}} V_{A B A^{\prime} B^{\prime}}^{p g}\left[\hat{\rho}_{B^{\prime} A}^{g p}, \hat{\rho}_{B A^{\prime}}^{p g}\right]_{+}+\hat{H}_{S},
\end{aligned}
$$

Here, $[C, D]_{+}=(1 / 2)(C D+D C)$.

Substituting Eqs. (2.8)-(2.10) to Eq. (2.3) and performing necessary commutation procedures, one obtains the following equation:

$$
\begin{aligned}
& i \hbar \frac{\partial \rho_{A B}^{p}(t)}{\partial t}=\sum_{C}\left[\left(\hat{H}_{p}\right)_{A C} \rho_{C B}^{p}(t)-\rho_{A C}^{p}(t)\left(\hat{H}_{p}\right)_{C B}\right]+ \\
& +\sum_{C}\left\{e_{p}\left[\left(\hat{\varphi}^{(S)}(t)\right)_{A C}, \rho_{C B}^{p}(t)\right]_{+}-e_{p}\left[\rho_{A C}^{p}(t),\left(\hat{\varphi}^{(S)}(t)\right)_{C B}\right]_{+}\right\}+ \\
& +\sum_{C} \sum_{A^{\prime} B^{\prime}}\left\{V_{A C B^{\prime} A^{\prime}}^{p g}\left[\rho_{A^{\prime} B^{\prime}}^{g}(t), \rho_{C B}^{p}(t)\right]_{+}-V_{C B A^{\prime} B^{\prime}}^{p g}\left[\rho_{A C}^{p}(t), \rho_{A^{\prime} B^{\prime}}^{g}(t)\right]_{+}\right\} .
\end{aligned}
$$

Transform the density matrix $\rho_{A B}^{p}(t)$ and external scattering potential $\varphi_{S}$ into the sum of averaged values and corresponding fluctuations:

$$
\begin{aligned}
& \rho_{A B}^{p}(t)=f_{A B}^{p}(t)+\delta \rho_{A B}^{p}(t) \\
& \left(\varphi_{S}(t)\right)_{A B}=\left\langle\left(\varphi_{S}(t)\right)_{A B}\right\rangle+\left(\delta \varphi_{S}(t)\right)_{A B} .
\end{aligned}
$$

Then, we obtain the following equation for the oneparticle density matrix $f_{A}^{p}=\left\langle\rho_{A A}^{p}\right\rangle$ :

$i \hbar \frac{\partial f_{A}^{p}}{\partial t}-\hat{Z}\left(f_{A}^{p}\right)=i \hbar \operatorname{St} f_{A}^{p}$

Here,

$$
\begin{aligned}
& \hat{\mathrm{Z}}\left(f_{A}^{p}\right)=\sum_{C}\left[\left(\hat{H}_{p}\right)_{A C} f_{C B}^{p}-\left(\hat{H}_{p}\right)_{C B} f_{A C}^{p}\right](\text { at } B \rightarrow A), \\
& \mathrm{St} f_{A}^{p}(t)=\mathrm{St}_{p-S} f_{A}^{p}(t)+\sum_{g} \mathrm{St}_{p g} f_{A}^{p}(t), \\
& \mathrm{St}_{p-S} f_{A}^{p}(t)=\left(e_{p} / i \hbar\right) \sum_{B}\left[\begin{array}{l}
\left.\left\langle\left(\delta \varphi_{S}(t)\right)_{A B}, \delta \rho_{B A}^{p}(t)\right)_{+}\right\rangle_{-} \\
-\left\langle\left(\left(\delta \varphi_{S}(t)\right)_{B A}, \delta \rho_{A B}^{p}(t)\right)_{+}\right\rangle
\end{array}\right], \\
& \mathrm{St}_{p g} f_{A}^{p}(t)=(1 / i \hbar) \sum_{g} \sum_{B A^{\prime} B^{\prime}}\left[\begin{array}{l}
V_{A B B^{\prime} A^{\prime}}^{p g}\left\langle\left(\delta \rho_{A^{\prime} B^{\prime}}^{g}(t), \delta \rho_{B A}^{p}(t)\right)_{+}\right\rangle_{-}^{p g} \\
-V_{B B^{\prime} A^{\prime}}^{p g}\left\langle\left(\delta \rho_{B^{\prime} A^{\prime}}^{g}(t), \delta \rho_{A B}^{p}(t)\right)_{+}\right\rangle
\end{array}\right]
\end{aligned}
$$




\section{Matrix elements of Hamiltonian $\mathbf{H}_{p}$}

In this article, for the following calculations we use the system of plane waves and accept the quantum number $A$ as the corresponding set of three components of wave vector $\vec{k}$ (and so on):

$$
\begin{aligned}
& A \rightarrow \vec{k}_{A}=\left(k_{A x}, k_{A y}, k_{A z}\right), \\
& \Psi_{A}(\vec{r})=\Psi_{\vec{k}_{A}}(\vec{r})=\prod_{w} \Psi\left(k_{A w} w\right)=\prod_{w} L^{-1 / 2} \exp \left(i k_{A w} w\right) .
\end{aligned}
$$

Here and farther, $w=x, y, z$ and $-L / 2 \leq w \leq L / 2$. We suppose that every linear dimension $L$ of the considered system exceeds utmost every characteristic length and tends to infinity. The functions $\Psi_{A}(\vec{r})$ are proper functions for the operator of momentum $\hat{\vec{p}}=\hbar \hat{\vec{k}}$ and for the operator of kinetic energy $\varepsilon^{p}(\overrightarrow{\hat{k}})$ :

$$
-i \nabla_{w} \Psi\left(k_{A w} ; w\right)=k_{A w} \Psi\left(k_{A w} ; w\right)
$$

and

$$
\hat{\mathcal{E}}^{p} \Psi_{\vec{k}}(\vec{r})=\varepsilon^{p}(\vec{k}) \Psi_{\vec{k}}(\vec{r}),
$$

where $\varepsilon^{p}(\vec{k})$ is dispersion law for $p$-carriers.

Eq. (2.6) shows that Hamiltonian $\hat{H}_{p}$ evidently depends on potential spatial coordinates. In spite of all points in the $\vec{r}$-space are equivalent, this Hamiltonian containing the field-dependent term $\hat{H}_{p}^{(E)}$ is not arbitrary invariant in space. Therefore, a specific problem appears for solution of this kinetic equation. Usually, when calculating the collision integral $\operatorname{St} f_{\vec{k}}^{p}$, the field term $\hat{H}^{(E)}$ is simply omitted in this collision integral (and we call that way, see, for instance, [2, 3] and [7-9], as the "standard variant"). In this paper, we also consider another one called as "non-standard variant" (see [11] and [15]), for which the field term $\left(\hat{H}^{(E)}\right)_{A C}$ in St $f_{\vec{k}}$ is retained. Below, inside the collision integral we use the following designation

$$
\left(\hat{H}_{e}\right)_{A B}=\left(\hat{H}^{(0)}\right)_{A B}+\chi\left(\hat{H}^{(E)}\right)_{A B} \text {. }
$$

Here,

$\chi=0$ for the standard variant,
Now, we take into consideration that functions (3.4) are invariant to the shift of argument $w$ on the de Broglie wavelength $\lambda_{A w}=2 \pi / k_{A w}$ :

$\Psi_{A}\left(w+\lambda_{A w}\right)=L^{-1 / 2} \exp \left[i k_{A w}\left(w+\lambda_{A w}\right)\right]=\Psi_{A}(w)$.

It is easy to convince that the matrix element of coordinate $w$ is proportional to the Kronecker symbol:

$(w)_{A B}=\int_{-L / 2}^{L / 2} w \Psi^{*}\left(k_{A w} ; w\right) \Psi\left(k_{B w} ; w\right) d w=(w)_{A A} \delta_{A, B}$

To calculate the value $(w)_{A A}$, we use in the formulae (3.9) the shifted space of integration $L_{A w}(-) \leq w \leq L_{A w}(+)$. Then, we obtain:

$(w)_{A A}=\int_{L_{A w}(-)}^{L_{A w}(+)} w \Psi^{*}\left(k_{A w} ; w\right) \Psi\left(k_{A w} ; w\right) d w$.

Here,

$$
L_{A w}(+)=L / 2+\lambda_{A w}, \quad L_{A w}(-)=-L / 2+\lambda_{A w} .
$$

As a result, we find:

$$
\begin{aligned}
& (w)_{A A}=\int_{L_{A w}(-)}^{L_{A w}(+)} w \Psi^{*}\left(k_{A w} ; w\right) \Psi\left(k_{A w} ; w\right) d w= \\
& =L^{-1} \int_{L_{A w}(-)}^{L_{A w}(+)} w d w=\lambda_{A w}=2 \pi / k_{A w}, \\
& (w)_{A B}=\left(2 \pi / k_{A w}\right) \delta_{A B} .
\end{aligned}
$$

Represent the matrix element of Hamiltonian $\hat{H}_{p}$ by the following form (see (3.5)):

$$
\left(\hat{H}_{p}\right)_{A B}=\bar{\varepsilon}_{A}^{p} \delta_{A, B}=\hbar \bar{\omega}_{A}^{p} \delta_{A, B}=\left(\varepsilon_{A}^{p}-\chi \frac{2 \pi e_{p} E_{z}}{k_{z}}\right) \delta_{A, B} .
$$

Using the designations $\vec{k}_{A} \rightarrow \vec{k}$ and $\vec{k}_{B} \rightarrow \vec{k}-\vec{q}$, one obtains the formulae

$\bar{\varepsilon}_{A B}^{p}=\bar{\varepsilon}_{A}^{p}-\bar{\varepsilon}_{B}^{p} \rightarrow \bar{\varepsilon}_{\vec{k}}^{p}-\bar{\varepsilon}_{\vec{k}-\vec{q}}^{p}=\varepsilon_{\vec{k}}^{p}-\varepsilon_{\vec{k}-\vec{q}}^{p}+\chi \Theta^{p}(\vec{k}, \vec{k}-\vec{q})$, 
$\Theta^{p}(\vec{k}, \vec{k}-\vec{q})=2 \pi e_{p} E_{z} q_{z} / k_{z}\left(k_{z}-q_{z}\right)$.

Farther, in this article we accept for $p$-carriers the dispersion law of the following form (see, for instance, the formulae (7.3)):

$\omega_{\vec{k}}^{p}=\varepsilon_{\vec{k}}^{p} / \hbar=(\hbar / 2 m) \vec{k} \tilde{D}^{p} \vec{k}, \vec{E}=\left(0,0, E_{z}\right)$.

Let us introduce the new designations:

$\tilde{D}^{p}=\tilde{B}^{p} \cdot \tilde{B}^{p}, \vec{\kappa}=\vec{k} \tilde{B}^{p}, \vec{Q}^{p}=\vec{q} \tilde{B}^{p}$.

For small vectors $\vec{q}$, farther we use the approximated form:

$\Theta^{p}(\vec{k}, \vec{k}-\vec{q})=2 \pi e_{p} E_{z} q_{z} / k_{z}\left(k_{z}-q_{z}\right) \rightarrow$

$\rightarrow 2 \pi e_{p} E_{z} q_{z} / k_{z}^{2} \rightarrow 2 \pi e_{p} E_{z} q_{z} /\left\langle k_{z}^{2}\right\rangle=$

$=4 \pi e_{p} E_{z} B_{z z}^{p} Q_{z}^{p} \frac{\hbar^{2} F_{1 / 2}(\eta)}{m k_{B} T F_{3 / 2}(\eta)}=e_{p} E_{z} Q_{z}^{p} \hbar \xi^{p}$,

where $\eta=\varepsilon_{\mathrm{F}} / k_{\mathrm{B}} T$ and

$\xi^{p} \approx 4 \pi \hbar\left(D_{z z}^{p}\right)^{1 / 2} F_{1 / 2}(\eta) / m k_{\mathrm{B}} T F_{3 / 2}(\eta)$,

$F_{r}(\eta)=\frac{1}{\Gamma(r+1)} \int_{0}^{\infty} \frac{w^{r} d w}{1+\exp (w-\eta)}$.

In what follows, we will use the simplified form (see Eqs. (3.15)-(3.20))

$\bar{\omega}_{\vec{k}, \vec{k}-\vec{q}}^{p} \rightarrow \omega_{\vec{\kappa}}^{p}-\omega_{\vec{\kappa}-\vec{Q}}^{p}+\chi e_{p} \xi^{p} E_{z} Q_{z}^{p}$.

\section{Collision integrals}

When using the Laplace transformation (see [8])

$\zeta(\omega)=\int_{0}^{\infty} \zeta(t) \exp (i \omega t) d t$

$\zeta(t)=\frac{1}{2 \pi} \int_{-\infty+i 0}^{\infty+i 0} \zeta(\omega) \exp (-i \omega t) d \omega$

Eq. (2.12) accepts the form

$-i \hbar \delta \rho_{A B}^{p}(t=0)+\hbar \omega \delta \rho_{A B}^{p}(\omega)=\left(\varepsilon_{A}^{p}-\varepsilon_{B}^{p}\right) \delta \rho_{A B}^{p}(\omega)+$

$+\left(f_{B}^{p}-f_{A}^{p}\right)\left[e_{p}\left(\delta \varphi^{(S)}\right)_{A B}+\sum_{g} \sum_{A^{\prime} B^{\prime}} V_{A B B^{\prime} A^{\prime}}^{p g} \delta \rho_{A^{\prime} B^{\prime}}^{p g}(\omega)\right]$.

Introduce the following designations:

$\delta \rho_{A B}^{p(0)}(\omega)=\frac{i \rho_{A B}^{p}(t=0)}{\omega-\bar{\omega}_{A B}^{p}+i 0} ; \quad M_{A B}^{p}(\omega)=\frac{f_{B}^{p}-f_{A}^{p}}{\hbar\left(\omega-\bar{\omega}_{A B}^{p}+i 0\right)}$.
Then, we find the lowest term in the set of perturbations theory:

$$
\begin{aligned}
& \delta \rho_{A B}^{p}(\omega)=\delta \rho_{A B}^{p(0)}(\omega)+ \\
& +M_{A B}^{p}(\omega)\left[e_{p}\left(\delta \varphi^{(S)}(\omega)\right)_{A B}+\sum_{g} \sum_{A^{\prime} B^{\prime}} V_{A B B^{\prime} A^{\prime}}^{p g} \delta \rho_{A^{\prime} B^{\prime}}^{p g}(\omega)\right] .
\end{aligned}
$$

Due to uniformity of time, the correlator of fluctuations $\delta \varphi^{(S)}(\omega)$ can be written as

$$
\begin{aligned}
& \left\langle\delta \varphi_{A B}^{(S)}(\omega), \delta \varphi_{B A}^{(S)}\left(\omega^{\prime}\right)\right\rangle=2 \pi \delta\left(\omega+\omega^{\prime}\right)\left\langle\delta \varphi_{(S)}^{2}\right\rangle_{\omega}^{A B}= \\
& =\delta_{A B}(2 \pi)^{-2} \delta\left(\omega+\omega^{\prime}\right) \int d^{3} \vec{q}\left|\left(b_{\vec{q}}\right)_{A B}\right|^{2}\left\langle\delta \varphi_{(S)}^{2}\right\rangle_{\omega, \vec{q}}
\end{aligned}
$$

Here,

$$
\left(b_{\vec{q}}\right)_{A B}=\int \Psi_{A}^{*}(\vec{r}) \exp (i \vec{q} \vec{r}) \Psi_{B}(\vec{r}) d^{3} \vec{r}
$$

In the second order of simplified theory of perturbation (see, for instance, [5])

$$
\left\langle\delta \rho_{A B}^{p}(t=0) \delta \rho_{A^{\prime} B^{\prime}}^{g}(t=0)\right\rangle \rightarrow \delta_{A^{\prime} B} \delta_{A B^{\prime}} \delta_{p g} f_{B}^{p}\left(1-f_{A}^{p}\right) .
$$

As a result, the collision integral for an equilibrium external scattering system has the form $\left(A \rightarrow \vec{k}_{A}\right.$ and $B \rightarrow \vec{k}_{B}$ )

$$
\begin{aligned}
& \mathrm{St}_{p-S} f_{A}^{p}=-\frac{e^{2}}{8 \pi^{3} \hbar^{2}} \int d^{3} \vec{k}_{B}\left\langle\delta \varphi_{(S)}^{2}\right\rangle_{\bar{\omega}_{A B}^{p}, \vec{k}_{A}-\vec{k}_{B}} . \\
& \cdot\left\{\left[f_{A}^{p}\left(1-f_{B}^{p}\right)+f_{B}^{p}\left(1-f_{A}^{p}\right)\right] \tanh \left(\frac{\hbar \bar{\omega}_{A B}^{p}}{2 \mathrm{k}_{\mathrm{B}} T}\right)+f_{A}^{p}-f_{B}^{p}\right\} .
\end{aligned}
$$

For the elastic scattering

$\left\langle\delta \varphi_{(S)}^{2}\right\rangle_{\bar{\omega}_{A B}^{p}, \vec{k}_{A}-\vec{k}_{B}}=\left\langle\delta \varphi_{(S)}^{2}\right\rangle_{\vec{k}_{A}-\vec{k}_{B}} \delta\left(\bar{\omega}_{\vec{k}_{A}, \vec{k}_{B}}^{p}\right)$,

then it follows from Eq. (4.7):

$$
\begin{aligned}
& \operatorname{St}_{p-S}\left(f_{A}^{p}\right)= \\
& =-\frac{e^{2}}{8 \pi^{3} \hbar^{2}} \int d^{3} \vec{k}_{B}\left(f_{A}^{p}-f_{B}^{p}\right)\left\langle\delta \varphi_{(S)}^{2}\right\rangle_{\vec{k}_{A}-\vec{k}_{B}} \delta\left(\bar{\omega}_{\vec{k}_{A}, \vec{k}_{B}}^{p}\right) .
\end{aligned}
$$

The collision integral for $p$-g-scattering can be presented by the form 
$\mathrm{St}_{p g} f_{A}^{p}=\frac{4 e^{4}}{\hbar^{2} L^{3}} \sum_{B A^{\prime} B^{\prime}} \int \frac{d^{3} \vec{q}}{q^{4}\left|\varepsilon\left(\bar{\omega}_{A B}^{p}, \vec{q}\right)\right|^{2}}\left|\left(b_{\vec{q}}\right)_{A B}\right|^{2}\left|\left(b_{\vec{q}}\right)_{A^{\prime} B^{\prime}}\right|^{2} \times$ $\times \delta\left(\bar{\omega}_{A B}^{p}-\bar{\omega}_{A^{\prime} B^{\prime}}^{g}\right) \times$

$\times\left[f_{B}^{p}\left(1-f_{A}^{p}\right) f_{A^{\prime}}^{g}\left(1-f_{B^{\prime}}^{g}\right)-f_{A}^{p}\left(1-f_{B}^{p}\right) f_{B^{\prime}}^{g}\left(1-f_{A^{\prime}}^{g}\right)\right]$.

Now show the several correlators for different external scattering potentials (see Refs. [5, 6]). They have the simple forms

$$
\left\langle\delta \varphi_{(S)}^{2}\right\rangle_{\vec{q}}=\Phi_{(S)}(q) / q^{v(S)}
$$

For the system of charged impurities with the concentration $n_{I}$ (used here is the simplified dielectric function: $\left.1 / \varepsilon_{L}\left(\bar{\omega}_{A B}^{p}, \vec{q}\right) \rightarrow\left(1 / \varepsilon_{L}\right) \vartheta\left(q-q_{0}\right)[13,15]\right)$

$\Phi_{(C I)}(q)=\Phi_{(C I)} \vartheta\left(q-q_{0}\right)=32 \pi^{3} e^{2} n_{C I} \vartheta\left(q-q_{0}\right) / \varepsilon_{L}^{2}$,

$v(C I)=4$

(here, $n_{C I}$ is the density of charged centers).

For the piezoelectric scattering by longitudinal acoustic phonons

$\Phi_{(\Pi)}(q)=\Phi_{(\Pi)}=G k_{\mathrm{B}} T, v(\Pi)=2$,

at high temperatures $\left(\hbar \omega_{(p h)}<<k_{\mathrm{B}} T\right)$ for the quasielastic scattering by polar optical phonons one can use the expression

$\Phi_{(O p t)}(q)=\Phi_{(O p t)}=8 \pi^{2} k_{\mathrm{B}} T / \varepsilon^{*}, \quad v(O p t)=2$,

at the quasi-elastic scattering by acoustic phonons $\left(\hbar s q<<k_{\mathrm{B}} T\right)$

$\Phi_{(A c)}(q)=\Phi_{(A c)}=2 \pi E_{A}^{2} \mathrm{k}_{\mathrm{B}} T / e^{2} \rho s^{2}, v(A c)=0$,

for neutral impurities (see [13])

$\Phi_{(N I)}(q)=\Phi_{(N I)}=8 \pi^{5} e^{2} r_{\mathrm{B}}^{4} n_{(D)}^{(3)}\left[1+\exp \left(-\eta+\eta_{D}\right)\right]^{-1}, \quad v(N I)=0$

(here, $\eta=\varepsilon_{\mathrm{F}} / k_{\mathrm{B}} T, \eta_{D}=\varepsilon_{D} / k_{\mathrm{B}} T, \varepsilon_{\mathrm{F}}$ is Fermi energy, and the value $\varepsilon_{D}<0$ represents the energetic level of donor).

\section{Static kinetic equations}

This system has the form (see Eq. (2.12))

$$
\begin{array}{r}
\frac{e_{p}}{\hbar} \vec{E} \frac{\partial f_{\vec{k}}^{p}}{\partial \vec{k}}=\mathrm{St}_{p-S} f_{\vec{k}}^{p}+\sum_{g} \mathrm{St}_{p g} f_{\vec{k}}^{g}, \\
(p, g=1,2,3, \ldots)
\end{array}
$$

or (see also Eqs. (4.9) and (4.10))

$$
\frac{e_{p}}{\hbar} \vec{E} \frac{\partial f_{\vec{k}}^{p}}{\partial \vec{k}}=\frac{e^{2}}{8 \pi^{3} \hbar^{2}} P_{\vec{k}}^{p}(S)+\frac{e^{4}}{2 \pi^{3} \hbar^{2} \varepsilon_{L}^{2}} \sum_{g} W_{\vec{k}}^{p g} .
$$

Here (see (4.10), (4.11), (3.20)),

$$
\begin{aligned}
& P_{\vec{k}}^{p}(S)=-\int d^{3} \vec{k}^{\prime}\left(f_{\vec{k}}^{p}-f_{\vec{k}^{\prime}}^{p}\right)\left\langle\delta \varphi_{(S)}^{2}\right\rangle_{\vec{k}-\vec{k}^{\prime}} \delta\left(\bar{\omega}_{\vec{k}^{\prime}, \vec{k}^{\prime}}^{p}\right)= \\
& \left.=\int d^{3} \vec{q}\left(f_{k}^{p}-f_{\vec{k}-\bar{q}}^{p}\right)\left\langle\delta \varphi_{(S)}^{2}\right\rangle\right\rangle_{q} \delta\left(\bar{\omega}_{\vec{k}, \vec{k}-\vec{q}}^{p}\right) ; \\
& W_{\vec{k}}^{p g}=\int d^{3} \vec{k}^{\prime} \int \frac{d^{3} \vec{q}}{q^{4}} \vartheta\left(q-q_{0}\right) \delta\left(\bar{\Omega}_{p g}\left(\vec{k}, \vec{k}^{\prime}, \vec{q}\right)\right) \Lambda_{p g}\left(\vec{k}, \vec{k}^{\prime}, \vec{q}\right),
\end{aligned}
$$

where (see (3.17))

$$
\begin{aligned}
& \bar{\Omega}^{p g}\left(\vec{k}, \vec{k}^{\prime}, \vec{q}\right)=\bar{\omega}_{\vec{k}, \vec{k}-\vec{q}}^{p}+\bar{\omega}_{\vec{k}^{\prime}, \vec{k}^{\prime}+\vec{q}}^{g}= \\
& =\Omega^{p g}\left(\vec{k}, \vec{k}^{\prime}, \vec{q}\right)+\chi \hbar \xi \vec{E}\left(e_{p} \tilde{D}^{p}-e_{g} \tilde{D}^{g}\right) \vec{q},
\end{aligned}
$$

$\Omega^{p g}\left(\vec{k}, \vec{k}^{\prime}, \vec{q}\right)=\omega_{\vec{k}, \vec{k}-\vec{q}}^{p}+\omega_{\vec{k}^{\prime}, \vec{k}^{\prime}+\vec{q}}^{g}=$

$$
=(\hbar / m)\left[\left(\vec{k} \tilde{D}^{p}-\vec{k}^{\prime} \tilde{D}^{g}\right) \vec{q}-\vec{q}\left(\tilde{D}^{p}+\tilde{D}^{g}\right) \vec{q} / 2\right],
$$

$\bar{\Omega} p p\left(\vec{k}, \vec{k}^{\prime}, \vec{q}\right)=\Omega^{p p}\left(\vec{k}, \vec{k}^{\prime}, \vec{q}\right)=$

$=(\hbar / m)\left(\vec{k}-\vec{k}^{\prime}-\vec{q}\right) \tilde{D}^{p} \vec{q}$,

$$
\begin{aligned}
& \Lambda_{p g}\left(\vec{k}, \vec{k}^{\prime}, \vec{q}\right)=f_{\vec{k}-\vec{q}}^{p}\left(1-f_{\vec{k}}^{p}\right) f_{\vec{k}^{\prime}+\vec{q}}^{g}\left(1-f_{\vec{k}^{\prime}}^{s}\right)- \\
& -f_{\vec{k}}^{p}\left(1-f_{\vec{k}-\vec{q}}^{p}\right) f_{\vec{k}^{\prime}}^{g}\left(1-f_{\vec{k}^{\prime}+\vec{q}}^{g}\right) .
\end{aligned}
$$

Now, let us separate the distribution function $f_{\vec{k}}^{p}$ and the function $\Lambda_{p g}\left(\vec{k}, \vec{k}^{\prime}, \vec{q}\right)$ by equilibrium and nonequilibrium terms (there $\eta=\varepsilon_{\mathrm{F}} / k_{\mathrm{B}} T$ ): 


$$
\begin{aligned}
& f_{\vec{k}}^{p}=f_{\vec{k}}^{0 p}+f_{\vec{k}}^{1 p}, \\
& f_{\vec{k}}^{0 p}=\left\{1+\exp \left[\left(\varepsilon_{\vec{k}}^{p} / k_{\mathrm{B}} T\right)-\eta\right]\right\}^{-1}=\left(1+C_{\vec{k}}^{p}\right)^{-1}, \\
& 1-f_{\vec{k}}^{0 p}=C_{\vec{k}}^{p}\left(1+C_{\vec{k}}^{p}\right)^{-1}, \\
& \Lambda_{p g}\left(\vec{k}, \vec{k}^{\prime}, \vec{q}\right)=\Lambda_{p g}^{(0)}\left(\vec{k}, \vec{k}^{\prime}, \vec{q}\right)+\Lambda_{p g}^{(1)}\left(\vec{k}, \vec{k}^{\prime}, \vec{q}\right), \\
& \Lambda_{p g}^{(0)}\left(\vec{k}, \vec{k}^{\prime}, \vec{q}\right)=f_{\vec{k}-\vec{q}}^{0 p}\left(1-f_{\vec{k}}^{0 p}\right) f_{\vec{k}^{\prime}+\vec{q}}^{0 g}\left(1-f_{\vec{k}^{\prime}}^{0 g}\right)- \\
& -f_{\vec{k}}^{0 p}\left(1-f_{\vec{k}-\vec{q}}^{0 p}\right) f_{\vec{k}^{\prime}}^{0 g}\left(1-f_{\vec{k}^{\prime}+\vec{q}}^{0 g}\right)= \\
& =\left(1+C_{k-q}^{p}\right)^{-1}\left(1+C_{k}^{p}\right)^{-1}\left(1+C_{k^{\prime}}^{g}\right)^{-1}\left(1+C_{k^{\prime}+q}^{g}\right)^{-1} \times \\
& \times C_{k}^{p} C_{k^{\prime}}^{g}\left(1-C_{k-q}^{p} C_{k^{\prime}+q}^{g} / C_{k}^{p} C_{k^{\prime}}^{g}\right)= \\
& \approx\left(k_{\mathrm{B}} T\right)^{2}\left(\partial f_{\mathrm{\kappa}}^{0(p)} / \partial \varepsilon_{\mathrm{\kappa}}^{p}\right)\left(\partial f_{\mathrm{\kappa}}^{0(g)} / \partial \varepsilon_{\kappa}^{g}\right) \times \\
& \times\left(1-C_{k-q}^{p} C_{\vec{k}^{\prime}+q}^{g} / C_{k}^{p} C_{k^{\prime}}^{g}\right), \\
& \Lambda_{p g}^{(1)}\left(\vec{k}, \vec{k}^{\prime}, \vec{q}\right)=f_{\vec{k}-\vec{q}}^{1 p}\left(1-f_{\vec{k}}^{0 p}\right) f_{\vec{k}^{\prime}+\vec{q}}^{0 g}\left(1-f_{\vec{k}^{\prime}}^{0 g}\right)- \\
& -f_{\vec{k}-\vec{q}}^{0 p} f_{\vec{k}}^{1 p} f_{\vec{k}^{\prime}+\vec{q}}^{0 g}\left(1-f_{\vec{k}^{\prime}}^{0 g}\right)+f_{\vec{k}-\vec{q}}^{0 p}\left(1-f_{\vec{k}}^{0 p}\right) f_{\vec{k}^{\prime}+\vec{q}}^{1 g}\left(1-f_{\vec{k}^{\prime}}^{0 g}\right)- \\
& f_{\vec{k}-\vec{q}}^{0 p}\left(1-f_{\vec{k}}^{0 p}\right) f_{\vec{k}^{\prime}+\vec{q}}^{0 g} f_{\vec{k}^{\prime}}^{1 g}-f_{\vec{k}}^{1 p}\left(1-f_{\vec{k}-\vec{q}}^{0 p}\right) f_{\vec{k}^{\prime}}^{0 g}\left(1-f_{\vec{k}^{\prime}+\vec{q}}^{0 g}\right)+ \\
& +f_{\vec{k}}^{0 p} f_{\vec{k}-\vec{q}}^{1 p} f_{\vec{k}^{\prime}}^{0 g}\left(1-f_{\vec{k}^{\prime}+\vec{q}}^{0 g}\right)-f_{\vec{k}}^{0 p}\left(1-f_{\vec{k}-\vec{q}}^{0 p}\right) f_{\vec{k}^{\prime}}^{1 g}\left(1-f_{\vec{k}^{\prime}+\vec{q}}^{0 g}\right)+ \\
& +f_{\vec{k}}^{0 p}\left(1-f_{\vec{k}-\vec{q}}^{0 p}\right) f_{\vec{k}^{\prime}}^{0 g} f_{\vec{k}^{\prime}+\vec{q}}^{1 g} \cdot \\
& (5.12) \\
& \\
& (19.11) \\
&
\end{aligned}
$$

Below, one supposes the non-equilibrium function $f_{\vec{k}}^{1 p}$ as small amendment to the equilibrium function $f_{\vec{k}}^{0 p}$. Farther our investigation will be restricted by linear terms (relatively to the external electrical field $E$ only). In this paper, we use the following model for $f_{\vec{k}}^{1 p}$ (see (5.9)):

$$
\begin{aligned}
& f_{\vec{k}}^{1 p}=e_{p} \vec{E} \tilde{D}^{p} \vec{k} C_{\vec{k}}^{p}\left(1+C_{\vec{k}}^{p}\right)^{-2} R\left(\varepsilon_{\vec{k}}^{p}\right) / k_{\mathrm{B}} T= \\
& =e_{p} \vec{E} \tilde{D}^{p} \vec{k} R\left(\varepsilon_{\vec{k}}^{p}\right)\left(-\partial f_{\vec{k}}^{0 p} / \partial \varepsilon_{\vec{k}}^{p}\right) .
\end{aligned}
$$

Here, $R\left(\varepsilon_{\vec{k}}^{p}\right)$ is the model unknown function that will be find later. Then (see Eqs. (3.20), (4.11), (5.3))

$$
\begin{aligned}
& \delta\left(\bar{\omega}_{\vec{k}, \vec{k}-\vec{q}}^{p}\right)\left(f_{\vec{k}}^{p}-f_{\vec{k}-\vec{q}}^{p}\right)= \\
& =\hbar \delta\left[\varepsilon_{\vec{k}}^{p}-\varepsilon_{\vec{k}-\vec{q}}^{p}+\chi \hbar e_{p} \xi \vec{E} \widetilde{D}^{p} \vec{q}\right] \times \\
& \times\left(f_{\vec{k}}^{0 p}-f_{\vec{k}-\vec{q}}^{0 p}+f_{\vec{k}}^{1 p}-f_{\vec{k}-\vec{q}}^{1 p}\right) \rightarrow \\
& \rightarrow \delta\left(\omega_{\vec{k}, \vec{k}-\vec{q}}^{p}\right)\left(\partial f_{\vec{k}}^{0 p} / \partial \varepsilon_{\vec{k}}^{p}\right)\left(\chi \xi \hbar-R\left(\varepsilon_{\vec{k}}^{p}\right)\right] e_{p} \vec{E} \tilde{D}^{p} \vec{q} .
\end{aligned}
$$

Now, the coefficient $P_{\vec{k}}^{p}(S)$ accepts the form (see $(4.11)-(4.16))$

$P_{\vec{k}}^{p}(S)=\frac{2 m}{\hbar}\left(-\partial f_{\vec{k}}^{0 p} / \partial \varepsilon_{\vec{k}}^{p} / \partial \varepsilon_{\vec{k}}^{p}\right)\left[R\left(\varepsilon_{\vec{k}}^{p}\right)-\chi \xi \hbar\right] e_{p} \vec{E} \tilde{D}^{p} \times$ $\times \int \Phi_{(S)}(q) q^{-v(S)} \vec{q} \delta\left[\vec{k} \tilde{D}^{p} \vec{k}-(\vec{k}-\vec{q}) \tilde{D}^{p}(\vec{k}-\vec{q})\right] d^{3} \vec{q}$.

Farther, let us consider the matrix $\tilde{D}^{p}$ to be diagonal; try also to approximate the integral from the right part of the expression (5.15). Then, the result is as follows (see also the forms (3.17), (3.18) and (7.3a)):

$$
\begin{aligned}
& \int \Phi_{(S)}(q) q^{-v(S)} \vec{q} \delta\left[\vec{k} \widetilde{D}^{p} \vec{k}-(\vec{k}-\vec{q}) \tilde{D}^{p}(\vec{k}-\vec{q})\right] d^{3} \vec{q}= \\
& =\tilde{M}_{(S)}^{p} \vec{\kappa} r_{(S)}(\kappa) \Phi_{(S)} .
\end{aligned}
$$

Here,

$$
\begin{aligned}
& \left.\tilde{M}_{(S)}^{p} \approx\left|\tilde{D}_{p}\right|^{-1 / 2}\left[3 / \operatorname{Spur}\left(\tilde{D}_{p}^{-1}\right)\right]\right]^{\nu(S) / 2} \tilde{B}_{p}^{-1}, \\
& r_{(C I)}(\kappa)=\pi Y / \kappa^{3}, \\
& r_{(S \neq C I)}(\kappa)=\pi(2)^{4-v(S)} \kappa^{1-v(S)} /[4-v(S)], \\
& Y \approx(1 / 2) \ln \left[4 \varepsilon_{L} \sqrt{8 k_{\mathrm{B}} T} \hbar F_{1}^{2}(\eta) / e^{2} \sqrt{m \pi} F_{1 / 2}^{2}(\eta) F_{-1 / 2}(\eta)\right] .
\end{aligned}
$$

Note that $\tilde{M}_{(S)}^{p}=\tilde{1}$ at $\tilde{D}_{p}=\tilde{1}$, the value

$$
Y \approx(1 / 2) \ln \left[4 \varepsilon_{L} \sqrt{8 k_{\mathrm{B}} T} \hbar F_{1}^{2}(\eta) / e^{2} \sqrt{m \pi} F_{1 / 2}^{2}(\eta) F_{-1 / 2}(\eta)\right]
$$

is the natural logarithm for the ratio of averaged deBroigle wavelength to screen length.

For $\vec{E}=\left(0,0, E_{z}\right)$ it follows from (5.9) and (5.15) $-(5.18)$ :

$$
\begin{aligned}
& P_{\overrightarrow{\mathrm{\kappa}}}^{p}(C I)=\left(2 m Y \pi \Phi_{(C I)} / \hbar\right)\left(-\partial f_{\vec{\kappa}}^{0(p)} / \partial \varepsilon_{\overrightarrow{\mathrm{\kappa}}}^{(p)}\right) \times \\
& \times\left[R\left(\varepsilon_{\overrightarrow{\mathrm{\kappa}}}^{(p)}\right)-\chi \xi \hbar\right] e_{p} E_{z} D_{z z}^{p} \kappa_{z}^{(p)} /\left(\kappa^{(p)}\right)^{3}, \\
& P_{\overrightarrow{\mathrm{\kappa}}}^{p}(S \neq C I)=\left(2 m \pi \Phi_{(S)} / \hbar\right)\left(-\partial f_{\overrightarrow{\mathrm{\kappa}}}^{0(p)} / \partial \varepsilon_{\overrightarrow{\mathrm{\kappa}}}^{(p)}\right) \times \\
& \times\left[R\left(\varepsilon_{\overrightarrow{\mathrm{\kappa}}}^{(p)}\right)-\chi \xi \hbar\right] e_{p} E_{z} D_{z z}^{p} \kappa_{z}^{(p)}(2)^{4-v(S)} \times \\
& \times\left(\kappa^{(p)}\right)^{1-v(S)} /[4-v(S)] .
\end{aligned}
$$


Now construct the other linear product:

$\delta\left(\vec{\Omega}_{p g}\left(\vec{k}, \vec{k}^{\prime}, \vec{q}\right)\right) \Lambda_{p g}\left(\vec{k}, \vec{k}^{\prime}, \vec{q}\right)=$

$\left.=\delta\left(\Omega^{p g}\left(\vec{k}, \vec{k}^{\prime}, \vec{q}\right)+\chi \xi \vec{E}\left(e_{p} \tilde{D}^{p}-e_{g} \tilde{D}^{g}\right) \vec{q}\right)\right) \times$

$\times\left[\Lambda_{p g}^{0}\left(\vec{k}, \vec{k}^{\prime}, \vec{q}\right)+\Lambda_{p g}^{1}\left(\vec{k}, \vec{k}^{\prime}, \vec{q}\right)\right] \approx$

$\approx \delta\left(\Omega^{p g}\left(\vec{k}, \vec{k}^{\prime}, \vec{q}\right)\right) \bar{\Lambda}_{p g}\left(\vec{k}, \vec{k}^{\prime}, \vec{q}\right)=\delta\left(\Omega^{p g}\left(\vec{k}, \vec{k}^{\prime}, \vec{q}\right)\right) \times$

$\times\left(\partial f_{\vec{k}}^{0 p} / \partial \varepsilon_{\vec{k}}^{p}\right)\left(\partial f_{\vec{k}^{\prime}}^{0 g} / \partial \varepsilon_{\vec{k}^{\prime}}^{g}\right) k_{\mathrm{B}} T \times$

$\times\left\{e_{g} \vec{E} \tilde{D}^{g} \vec{q}\left[R\left(\varepsilon_{\vec{k}}^{g}\right)-\chi \hbar \xi\right]-e_{p} \vec{E} \tilde{D}^{p} \vec{q}\left[R\left(\varepsilon_{\vec{k}}^{p}\right)-\chi \hbar \xi\right]\right\}$.

Further, we put for simplicity

$W_{\vec{k}}^{p g} \rightarrow W_{\overrightarrow{\mathrm{\kappa}}}^{p g}=\frac{m}{\hbar k_{\mathrm{B}} T}\left(1+C_{\overrightarrow{\mathrm{\kappa}}}^{p}\right)^{-2}\left(R\left(\varepsilon_{\overrightarrow{\mathrm{\kappa}}}^{p}\right)-\chi \xi \hbar\right) \times$

$\times C_{\overrightarrow{\mathrm{\kappa}}}^{p} e_{p} \vec{E}\left(\tilde{D}^{g}-\tilde{D}^{p}\right) \int d^{3} \vec{k}^{\prime}\left(1+C_{\overrightarrow{\mathrm{K}}^{\prime}}^{g}\right)^{-2} C_{\overrightarrow{\mathrm{K}}^{\prime}}^{g} \times$

$\times \int \vec{q} \frac{d^{3} \vec{q}}{q^{4}} \vartheta\left(q-q_{0}\right) \delta\left[\left(\vec{k} \tilde{D}^{p}-\vec{k}^{\prime} \tilde{D}^{g}\right) \vec{q}-\vec{q}\left(\tilde{D}^{p}+\tilde{D}^{g}\right) \vec{q} / 2\right] \approx$

$\approx \frac{2 \pi m}{\hbar} \zeta_{(W)}\left(\tilde{D}^{p}, \tilde{D}^{g}\right) F_{-1 / 2}(\eta) Y\left[-\partial f_{\vec{\kappa}}^{0 p} / \partial \varepsilon_{\vec{\kappa}}^{p}\right]\left[R\left(\varepsilon_{\vec{\kappa}}^{p}\right)-\chi \xi \hbar\right] \times$

$\times e_{p} E_{z}\left(D_{z z}^{g}-D_{z z}^{p}\right) \kappa_{z}\left(\left(2 m k_{\mathrm{B}} T\right) / \hbar^{2} \kappa^{2}\right)^{3 / 2}$.

Here, $\varsigma_{(W)}\left(\tilde{D}^{p}, \tilde{D}^{g}\right)=1$ if $\tilde{D}^{p}=\tilde{D}^{g}=1$.

Farther, we use the approximated expression:

$$
\begin{aligned}
& W_{\overrightarrow{\mathrm{\kappa}}}^{p g}=(2 \pi m / \hbar) F_{-1 / 2}(\eta) Y\left[-\partial f_{\overrightarrow{\mathrm{\kappa}}}^{0 p} / \partial \varepsilon_{\overrightarrow{\mathrm{\kappa}}}^{p}\right]\left[R\left(\varepsilon_{\overrightarrow{\mathrm{\kappa}}}^{p}\right)-\chi \xi \hbar\right] \times \\
& \times e_{p} E_{z}\left(D_{z z}^{g}-D_{z z}^{p}\right) \kappa_{z}\left(2 m k_{\mathrm{B}} T / \hbar^{2} \kappa^{2}\right)^{3 / 2} .
\end{aligned}
$$

Note that

$$
W_{\overrightarrow{\mathrm{\kappa}}}^{p p}=0 \text {, }
$$

therefore,

$\mathrm{St}_{p p} f_{\vec{k}}^{p}=0$

For the linear equation, one obtains with account of the expressions (5.20), (5.21) and (5.24) the equation for unknown function $R\left(\varepsilon_{\overrightarrow{\mathrm{\kappa}}}^{p}\right)$ :

$$
\begin{aligned}
& \frac{e_{p}}{\hbar \kappa} E_{z} \kappa_{z}\left(D_{z z}^{p}\right)^{1 / 2} \frac{\partial f_{\kappa}^{(0) p}}{\partial \kappa}=\frac{e^{2}}{8 \pi^{3} \hbar^{2}} P_{\bar{\kappa}}^{p}(S)+ \\
& +\frac{e^{4}}{2 \pi^{3} \hbar^{2} \varepsilon_{L}^{2}} \sum_{g} W_{\vec{\kappa}}^{p g} \equiv \frac{e^{2}}{8 \pi^{3} \hbar^{2}} \bar{P}_{\vec{\kappa}}^{p}(S) .
\end{aligned}
$$

\section{Solution of kinetic equation for simple system of carriers}

To solve the kinetic equation, one should find the nonequilibrium distribution function $f_{\vec{k}}^{1 p}$. At the adopted model of that (see (5.13)), there is the basic necessity to find a function $R(\varepsilon)$.

We shall consider in this section the solely system of band carriers $\left(f_{\vec{k}}^{p} \rightarrow f_{\vec{k}}\right)$ with the simple dispersion law (see (3.17)). Due to the expression (5.24), the system of equations (5.1) reduces to the only kinetic equation (our consideration is limited by the first degree of external electric field)

$\frac{e}{\hbar} \vec{E} \frac{\partial f_{\vec{k}}^{(0)}}{\partial \vec{k}}=\mathrm{St}_{e-S} f_{\vec{k}}$

or (for the case $S \rightarrow C I$ )

$\frac{e}{\hbar \kappa} E_{z} \kappa_{z}\left(D_{z z}\right)^{1 / 2} \frac{\partial f_{\kappa}^{(0)}}{\partial \kappa}=\frac{e^{2}}{8 \pi^{3} \hbar^{2}} P_{\vec{\kappa}}(S)$.

Introducing here the forms (5.19) and (5.21), one obtains:

$R_{(C I)}\left(\varepsilon_{\vec{\kappa}}\right)-\chi \xi \hbar=-\frac{4 \pi^{2} \hbar^{4} \kappa^{3}}{e^{2} m^{2} \Phi_{(C I)} Y\left(D_{z z}\right)^{1 / 2}}=$
$=-\frac{\hbar^{4} \varepsilon_{L}^{2} \kappa^{3}}{8 \pi e^{4} m^{2} n_{(C I)} Y\left(D_{z z}\right)^{1 / 2}}$,

$R_{(S \neq C I)}\left(\varepsilon_{\overrightarrow{\mathrm{\kappa}}}\right)-\chi \xi \hbar=$

$=-\frac{4 \pi^{2} \hbar^{4} \kappa^{v(S)-1}}{2^{4-v(S)} e^{2} m^{2}\left(D_{z z}\right)^{1 / 2} \Phi_{(S \neq C I)}} \rightarrow$

$\rightarrow-\frac{\hbar^{4} \varepsilon_{L}^{2} \kappa^{3}}{8 \pi e^{4} m^{2} n_{(S \neq C I)}\left(D_{z z}\right)^{1 / 2}}$.

Here we have introduced the concentration of "conditional scattering centers":

$$
\begin{aligned}
& n_{(S \neq C I)}(\kappa)=\Phi_{(S)} \varepsilon_{L}^{2} \kappa^{4-v(S \neq C I)} / 2^{1+v(S \neq C I)} \pi^{3} e^{2} \times \\
& \times[4-v(S \neq C I)] .
\end{aligned}
$$

Then one obtains the non-equilibrium part of the distribution function with the help of expressions (3.20), (4.12), (5.13), (6.3) and (6.4): 


$$
\begin{aligned}
& f_{\overrightarrow{\mathrm{\kappa}}}^{1}(C I) \approx-\frac{\pi \hbar^{2} D_{z z} e E_{z} \kappa_{z} F_{1 / 2}(\eta)}{m\left(k_{\mathrm{B}} T\right)^{2} F_{3 / 2}(\eta) \cosh ^{2}\left[\left(\varepsilon_{\overrightarrow{\mathrm{\kappa}}} / k_{\mathrm{B}} T\right)-\eta / 2\right]} \times \\
& \times\left[\frac{\hbar^{2} k_{\mathrm{B}} T \kappa^{3} \varepsilon_{L}^{2} F_{3 / 2}(\eta)}{32 \pi^{2} e^{4} m n_{(C I)} Y D_{z z}^{5 / 2} F_{1 / 2}(\eta)}-\chi\right] \equiv \\
& \quad \equiv-\frac{\pi \hbar^{2} D_{z z} e E_{z} \kappa_{z} F_{1 / 2}(\eta)}{m\left(k_{\mathrm{B}} T\right)^{2} F_{3 / 2}(\eta) \cosh ^{2}\left[\left(\varepsilon_{\overrightarrow{\mathrm{\kappa}}} / k_{\mathrm{B}} T\right)-\eta / 2\right]} \times \\
& \times\left[\frac{\hbar^{2} k_{\mathrm{B}} T \kappa^{3} \varepsilon_{L}^{2} F_{3 / 2}(\eta)}{32 \pi^{2} e^{4} m \bar{n}_{(C I)} D_{z z}^{5 / 2} F_{1 / 2}(\eta)}-\chi\right],
\end{aligned}
$$$$
f_{\overline{\mathrm{k}}}^{1}(S \neq C I) \approx-\frac{\pi \hbar^{2} D_{z z} e E_{z} \kappa_{z} F_{1 / 2}(\eta)}{m\left(k_{\mathrm{B}} T\right)^{2} F_{3 / 2}(\eta) \cosh ^{2}\left[\left(\varepsilon_{\overrightarrow{\mathrm{k}}} / k_{\mathrm{B}} T\right)-\eta / 2\right]} \times
$$$$
\times\left[\frac{\hbar^{2} k_{\mathrm{B}} T \kappa^{3} \varepsilon_{L}^{2} F_{3 / 2}(\eta)}{32 \pi^{2} e^{4} m n_{(S \neq C l)}(\kappa) D_{z z}^{5 / 2} F_{1 / 2}(\eta)}-\chi\right],
$$

or for arbitrary mechanism of relaxation

$$
\begin{aligned}
& f_{\overrightarrow{\mathrm{\kappa}}}^{1}(S) \approx-\frac{\pi \hbar^{2} D_{z z} e E_{z} \kappa_{z} F_{1 / 2}(\eta)}{m\left(k_{\mathrm{B}} T\right)^{2} F_{3 / 2}(\eta) \cosh ^{2}\left[\left(\varepsilon_{\vec{\kappa}} / k_{\mathrm{B}} T\right)-\eta / 2\right]} \times \\
& \times\left[\frac{\hbar^{2} k_{\mathrm{B}} T \kappa^{3} \varepsilon_{L}^{2} F_{3 / 2}(\eta)}{32 \pi^{2} e^{4} m \bar{n}_{(S)}(\kappa) D_{z z}^{5 / 2} F_{1 / 2}(\eta)}-\chi\right] .
\end{aligned}
$$

\section{Solution of kinetic equations for the system of three ellipsoidal valleys}

As an example of complicated system of band charged carriers, we consider three valleys shown as ellipsoids in Figure. Transitions of carriers between distinct valleys are farther omitted.

Let us introduce the following designations:

$\varepsilon_{\vec{k}}^{p}=\hbar \omega_{\vec{k}}^{p}=\left(\hbar^{2} / 2 m_{\perp}\right)\left(\vec{k} \tilde{D}^{(p)} \vec{k}\right), m_{\perp} \rightarrow m$,

$\gamma=m_{\perp} / m_{\Downarrow}=m / m_{\Downarrow}$.

$\omega_{\vec{k}}^{p}=\frac{\hbar}{2 m} \vec{k} \widetilde{D}^{p} \vec{k}, \omega_{\vec{k}^{\prime}}^{p}=\frac{\hbar}{2 m_{\perp}} \vec{k}^{\prime} \widetilde{D}^{p} \vec{k}^{\prime}, e_{p} \rightarrow e$.

$$
\tilde{D}^{a}=\left(\begin{array}{lll}
1 & 0 & 0 \\
0 & 1 & 0 \\
0 & 0 & \gamma
\end{array}\right), \tilde{D}^{b}=\left(\begin{array}{lll}
1 & 0 & 0 \\
0 & \gamma & 0 \\
0 & 0 & 1
\end{array}\right), \quad \tilde{D}^{c}=\left(\begin{array}{lll}
\gamma & 0 & 0 \\
0 & 1 & 0 \\
0 & 0 & 1
\end{array}\right),
$$

$$
\begin{aligned}
& \tilde{D}^{a}-\tilde{D}^{b}=\tilde{D}^{a b}=\left(\begin{array}{ccc}
0 & 0 & 0 \\
0 & 1-\gamma & 0 \\
0 & 0 & \gamma-1
\end{array}\right), \\
& \tilde{D}^{a}-\tilde{D}^{c}=\tilde{D}^{a c}=\left(\begin{array}{ccc}
1-\gamma & 0 & 0 \\
0 & 0 & 0 \\
0 & 0 & \gamma-1
\end{array}\right), \\
& \tilde{D}^{b}-\tilde{D}^{c}=\tilde{D}^{b c}=\left(\begin{array}{ccc}
1-\gamma & 0 & 0 \\
0 & \gamma-1 & 0 \\
0 & 0 & 0
\end{array}\right) .
\end{aligned}
$$

Here,

$$
\begin{aligned}
& D^{a}=D^{b}=D^{c}=D=3 / \operatorname{Spur}\left(\tilde{D}_{p}^{-1}\right)=3 /\left(2+\gamma^{-1}\right), \\
& \left|D^{a}\right|=\left|D^{b}\right|=\left|D^{c}\right|=\gamma .
\end{aligned}
$$
(5.2)):

The system of kinetic equations is as follows (see

$\frac{e}{\hbar} \vec{E} \frac{\partial f_{\vec{k}}^{0 a}}{\partial \vec{k}}=\frac{e^{2}}{8 \pi^{3} \hbar^{2}} P_{\vec{k}}^{a}(S)+\frac{e^{4}\left(W_{\vec{k}}^{a b}+W_{\vec{k}}^{a c}\right)}{2 \pi^{3} \hbar^{2} \varepsilon_{L}^{2}}$,

$\frac{e}{\hbar} \vec{E} \frac{\partial f_{\vec{k}}^{0 b}}{\partial \vec{k}}=\frac{e^{2}}{8 \pi^{3} \hbar^{2}} P_{\vec{k}}^{b}(S)+\frac{e^{4}\left(W_{\vec{k}}^{b c}+W_{\vec{k}}^{b a}\right)}{2 \pi^{3} \hbar^{2} \varepsilon_{L}^{2}}$,

$\frac{e}{\hbar} \vec{E} \frac{\partial f_{\vec{k}}^{0 c}}{\partial \vec{k}}=\frac{e^{2}}{8 \pi^{3} \hbar^{2}} P_{\vec{k}}^{c}(S)+\frac{e^{4}\left(W_{\vec{k}}^{c b}+W_{\vec{k}}^{c a}\right)}{2 \pi^{3} \hbar^{2} \varepsilon_{L}^{2}}$.

Using the expressions shown in Sec. 5, we find now the factors $R\left(\varepsilon_{\vec{k}}^{p}\right)$, containing in non-equilibrium distribution functions $f_{\vec{k}}^{1 p}$ (see the form (5.13)).

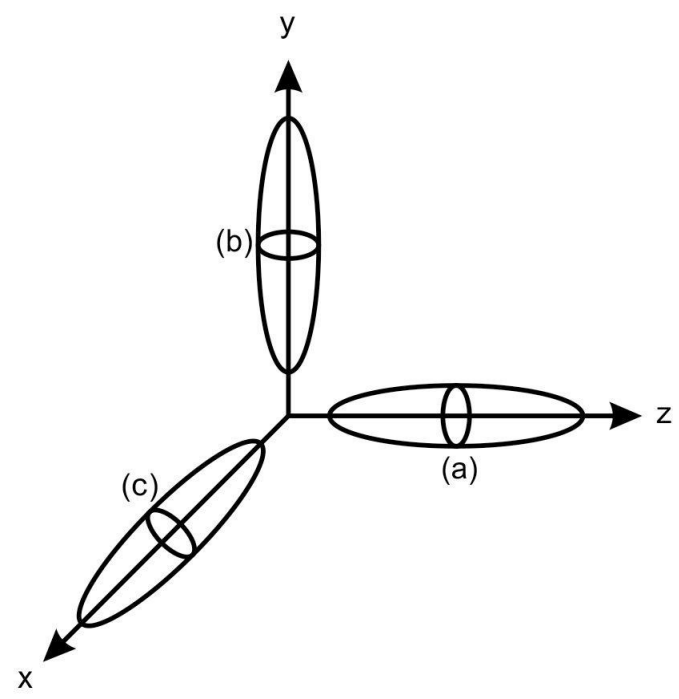

Fig. 1. 
Remember: $p \in a, b, c$ and $g \in a, b, c$. Below, we use the expression the concentration of carriers:

$$
n=\sum_{p} n^{(p)}=3 \gamma \frac{\left(k_{\mathrm{B}} T m\right)^{3 / 2}}{\sqrt{2} \pi^{3 / 2} \hbar^{3}} F_{1 / 2}(\eta)
$$

Inclosing the formulae (5.19) and (5.21) into the system (7.4)-(7.6), we obtain:

$$
\begin{aligned}
& P_{\vec{\kappa}}^{p}(S \neq C I)=\left[2 m \pi \Phi_{(S)} / \hbar(4-v(S))\right] \times \\
& \times\left(-\partial f_{\vec{\kappa}}^{0 p} / \partial \varepsilon_{\vec{\kappa}}^{p}\right)\left[R\left(\varepsilon_{\vec{\kappa}}^{p}\right)-\chi \xi \hbar\right] e_{p} E_{z} D_{z z}^{p} \kappa_{z}^{p}\left(\kappa^{p}\right)^{1-v(S)},
\end{aligned}
$$

$$
\begin{aligned}
& P_{\overrightarrow{\mathrm{\kappa}}}^{p}(S)=P_{\overrightarrow{\mathrm{\kappa}}}^{p}(C I)+\sum_{(S)} P_{\overrightarrow{\mathrm{\kappa}}}^{p}(S \neq C I)= \\
& =\left(-\partial f_{\overrightarrow{\mathrm{\kappa}}}^{0 p} / \partial \varepsilon_{\overrightarrow{\mathrm{\kappa}}}^{p}\right)\left[R\left(\varepsilon_{\overrightarrow{\mathrm{\kappa}}}^{p}\right)-\chi \xi \hbar\right] e_{p} E_{z} D_{z z}^{p} \kappa_{z}^{p} \times \\
& \times 64 e^{2} m \pi^{4}\left[Y n_{(C I)}+n^{*}\left(\kappa^{p}\right)\right] / \varepsilon_{L}^{2} \hbar\left(\kappa^{p}\right)^{3},
\end{aligned}
$$

where (see (6.5))

$$
n^{*}(\kappa)=\sum_{S \neq C I} n_{(S)}(\kappa)
$$

In what follows, we use the approximate expression (see (5.23))

$$
\begin{aligned}
& W^{p g}=\frac{2 \pi m}{\hbar} F_{-1 / 2}(\eta) Y\left[-\partial f_{\vec{\kappa}}^{0 p} / \partial \varepsilon_{\vec{\kappa}}^{p}\right] \times \\
& \times\left[R\left(\varepsilon_{\vec{\kappa}}^{p}\right)-\chi \xi \hbar\right] e_{p} E_{z}\left(D_{z z}^{g}-D_{z z}^{p}\right) \kappa_{z} \times \\
& \times\left(2 m k_{\mathrm{B}} T / \hbar^{2} \kappa^{2}\right)^{3 / 2} .
\end{aligned}
$$

Taking into attention the expressions (5.2), (5.23) and (7.7)-(7.11), one can obtain (see (6.5)-(6.7)):

$$
\begin{aligned}
& f_{\bar{\kappa}}^{1(p)}(C I) \approx-\frac{\pi \hbar^{2} D_{z z}^{p} e E_{z} \kappa_{z} F_{1 / 2}(\eta)}{m\left(k_{\mathrm{B}} T\right)^{2} F_{3 / 2}(\eta) \cosh ^{2}\left[\left(\varepsilon_{\overrightarrow{\mathrm{\kappa}}}^{p} / k_{\mathrm{B}} T\right)-\eta / 2\right]} \times \\
& \times\left[\frac{\hbar^{2} k_{\mathrm{B}} T\left(\kappa^{(p)}\right)^{3} \varepsilon_{L}^{2} F_{3 / 2}(\eta)}{32 \pi^{2} e^{4} m\left(Y n_{(C I)}+n^{*}\left(\kappa^{(p)}\right)+Y\left|\beta_{p}\right| n\right)\left(D_{z z}^{p}\right)^{5 / 2} F_{1 / 2}(\eta)}-\chi\right] .
\end{aligned}
$$

Here, $n$ is the total density of band carriers and (see (7.7) and (7.13))

$$
\beta_{p}=\sum_{g} \beta_{p g} \approx F_{-1 / 2}(\eta) \sum_{g}\left(D_{z z}^{g} / D_{z z}^{p}-1\right) / 6 \pi^{3 / 2} / F_{1 / 2}(\eta) .
$$

Note also that
$F_{-1 / 2}(-\eta>>1) / F_{1 / 2}(-\eta>>1) \rightarrow 1$,

$F_{-1 / 2}(\eta>>1) / F_{1 / 2}(\eta>>1) \rightarrow 2 / 3 \eta$,

$F_{1 / 2}(-\eta>>1) / F_{3 / 2}(-\eta>>1) \rightarrow 1$,

$F_{1 / 2}(\eta>>1) / F_{3 / 2}(\eta>>1) \rightarrow 5 / 2 \eta$.

If we consider mutual scattering of band electrons (for instance, $p$-carriers) and holes ( $g$-carriers), we have to change the form (7.13) to the expression

$$
\beta_{p} \approx F_{-1 / 2}(\eta) \sum_{g}\left(e_{g} D_{z z}^{g} / e_{p} D_{z z}^{p}-1\right) / 4 \pi^{3 / 2} / F_{1 / 2}(\eta) \text {. }
$$

\section{Other model of non-equilibrium distribution function}

Let us consider here the model of non-equilibrium distribution function in the form of Fermi-function with the "shifted" argument:

$f_{p}(\vec{k})=\left\{1+\exp \left[\varepsilon_{p}\left(\vec{k}-\vec{k}_{0}^{p}\right) / k_{\mathrm{B}} T-\eta\right]\right\}^{-1}$.

Here, the energy $\varepsilon_{\vec{k}}^{p}=\hbar^{2}\left(\vec{k} \tilde{D}^{p} \vec{k}\right) / 2 m=\hbar^{2}(\vec{\kappa})^{2} / 2 m$ and the velocity $\vec{v}^{p}=\hbar^{-1} \partial \varepsilon_{\vec{k}}^{p} / \partial \vec{k}=(\hbar / m) \vec{k} \tilde{D}^{p} \rightarrow(\hbar / m) \vec{\kappa} \widetilde{B}^{p}$.

The vector $\vec{k}_{0}^{p}$ is related with the drift velocity $\vec{v}_{0}^{p}$ by the relation

$\vec{k}_{0}^{p}=(m / \hbar) \tilde{D}_{p}^{-1} \vec{v}_{0}^{p}$

Then,

$\varepsilon_{p}\left(\vec{k}-\vec{k}_{0}^{p}\right)=\left(\hbar^{2} / 2 m\right)\left(\vec{k}-\vec{k}_{0}^{p}\right) \tilde{D}^{p}\left(\vec{k}-\vec{k}_{0}^{p}\right)=$

$=\left(\hbar^{2} / 2 m\right)\left[\kappa^{2}-(2 m / \hbar) \vec{\kappa} \tilde{B}_{p}^{-1} \vec{v}_{0}^{p}+(m / \hbar)^{2} \vec{v}_{0}^{p} \tilde{D}_{p}^{-1} \vec{v}_{0}^{p}\right]$.

After linearization over the velocity $\vec{v}_{0}^{p}$, one obtains

$$
\begin{aligned}
& f_{\vec{k}}^{1 p}=\hbar \vec{k} \vec{v}^{0(p)}\left(-\partial f_{\vec{k}}^{0} / \partial \varepsilon_{\vec{k}}^{p}\right)=\hbar k_{z} v_{z}^{0(p)}\left(-\partial f_{\vec{k}}^{0} / \partial \varepsilon_{\vec{k}}^{p}\right)= \\
& =\frac{\hbar k_{z} v_{z}^{0(p)}}{4 k_{\mathrm{B}} T \cosh ^{2}\left(\varepsilon_{\vec{k}}^{p} / 2 k_{\mathrm{B}} T-\eta / 2\right)} .
\end{aligned}
$$

Let in this section $S=C I$. Then, comparing (8.4) and (5.13), we have to perform the following action (see (5.26)):

$$
\begin{aligned}
& \bar{P}_{\vec{k}}^{p}(C I)=64 m e_{p}^{2} \pi^{4}\left(-\partial f_{\vec{k}}^{0 p} / \partial \varepsilon_{\vec{k}}^{p}\right)\left(R_{v}^{p}-\chi \xi \hbar\right) \times \\
& \times e_{p} E_{z} D_{z z}^{p} k_{z}^{(p)} \pi^{4} Y\left(n_{(C I)}+\beta_{p} n\right) / \varepsilon_{L}^{2} \hbar\left(k^{(p)}\right)^{3},
\end{aligned}
$$


where

$$
R_{v}^{p}=v_{z}^{0(p)} \hbar / e_{p} E_{z} \equiv R_{v}^{p} .
$$

For the case considered here, the kinetic equation takes the following form:

$$
\begin{aligned}
& \hbar k_{z}^{(p)} v_{z}^{0(p)}(C I)\left(-\partial f_{\vec{k}}^{(0) p} / \partial \varepsilon_{\vec{k}}^{p}\right) \approx \\
& \approx e_{p} E_{z} k_{z}^{(p)}\left(-\partial f_{\vec{\kappa}}^{0 p} / \partial \varepsilon_{\vec{\kappa}}^{p}\right) \times \\
& \times\left\{-\chi \xi \hbar+D_{z z}^{p} \hbar^{4} \varepsilon_{L}^{2}\left(k^{(p)}\right)^{3} / 8 Y \pi m^{2} e^{4} \times\right. \\
& \left.\times\left[n_{C I}+n F_{-1 / 2}(\eta) \sum_{g}\left(D_{z z}^{g} / D_{z z}^{p}-1\right) / 3 \gamma F_{1 / 2}(\eta)\right]\right\} .
\end{aligned}
$$

The supplement of operator $\int k_{z} d^{3} \vec{k}$ to the equation (8.7) leads to (see also (3.20)):

$$
\begin{aligned}
& v_{z}^{0(p)}(C I)=e_{p} E_{z} \frac{m k_{\mathrm{B}} T F_{3 / 2}(\eta)}{2 \pi \hbar F_{1 / 2}(\eta)} \times \\
& \times\left\{\frac{D_{z z}^{p} \hbar^{2} \varepsilon_{L}^{2} k_{T}^{3} k_{\mathrm{B}} T}{2 Y \pi^{5 / 2} m e^{4}\left(n_{C I}+\beta_{p} n\right)} \frac{F_{2}(\eta) F_{3 / 2}(\eta)}{\left[F_{1 / 2}(\eta)\right]^{2}}-\chi\right\} .
\end{aligned}
$$

Here,

$$
k_{T}=2 \pi / \bar{\lambda}_{\mathrm{deBr}}=\left(2 m k_{\mathrm{B}} T / \hbar^{2}\right)^{1 / 2} .
$$

Introducing (8.8) into (8.4), one finds

$$
\begin{aligned}
& f_{\vec{k}}^{1 p}=\frac{k_{z} m F_{3 / 2}(\eta) e_{p} E_{z}}{8 \pi F_{1 / 2}(\eta) \cosh ^{2}\left(\varepsilon_{\vec{k}}^{p} / 2 k_{\mathrm{B}} T-\eta / 2\right)} \times \\
& \times\left\{\frac{D_{z z}^{p} \hbar^{2} \varepsilon_{L}^{2} k_{T}^{3} k_{\mathrm{B}} T}{2 Y \pi^{5 / 2} m e^{4}\left(n_{C I}+\beta_{p} n\right)} \frac{F_{2}(\eta) F_{3 / 2}(\eta)}{\left(F_{1 / 2}(\eta)\right)^{2}}-\chi\right\} .
\end{aligned}
$$

For $\chi=1$, it follows from (8.4), (8.8) and (8.9) that:

$$
\begin{aligned}
& f_{\vec{k}}^{1 p}=\frac{k_{z} m e_{p} E_{z} F_{3 / 2}(\eta)}{8 \pi F_{1 / 2}(\eta) \cosh ^{2}\left(\varepsilon_{\vec{k}}^{p} / 2 k_{\mathrm{B}} T-\eta / 2\right)} \times \\
& \times\left[\left(\frac{\left.\left.\bar{L}_{(C I)}^{(p)}\right)^{3}-1\right] .}{\bar{\lambda}_{d e B r}}\right)^{3}\right]
\end{aligned}
$$

Here, the value $\bar{\lambda}_{\mathrm{deBr}}$ is the averaged deBroglie wavelength and $\bar{L}_{(C I)}^{(p)}$ can be named as an averaged length of relaxation:
$\bar{L}_{(C I)}^{(p)}=\left[\frac{4 D_{z z}^{p} \sqrt{\pi} \hbar^{2} \varepsilon_{L}^{2} k_{\mathrm{B}} T}{Y m e^{4}\left(n_{C I}+\beta_{p} n\right)}\right]^{1 / 3}$.

Note that in the case $\bar{L}_{(C I)}^{(p)}=\bar{\lambda}_{\mathrm{deBr}}$ the nonequilibrium part $f_{\vec{k}}^{1 p}$ becomes zero and the distribution function $f_{\vec{k}}^{p}$ becomes equilibrium, i.e., $f_{\vec{k}}^{p}=f_{\vec{k}}^{0 p}$. So the relation $f_{\vec{k}}^{p} \approx f_{\vec{k}}^{0 p}$ can be considered as superresistance.

\section{Conclusion}

It has been shown that in crystals with one band valley the mutual scattering of carriers does not contribute to the distribution function. Practically, we find the same result for many-valley systems with not high concentration of band charged carriers $\left(n \leq n_{C I}\right)$ : interaction between these particles does not introduce any essential contribution into the non-equilibrium function of distribution $f_{\vec{k}}^{1}$. But quite another situation appears for inter-particle co-operation with many-valley band structure and with a great number of band carriers. In this case, for $\beta n>n_{C I}$ the scattering by charged impurities does not dominate noticeably in comparison with the mutual scattering of band carriers.

Note also that the non-equilibrium distribution function has the traditional form at great ratio of relaxation length to average deBroglie wavelength (in this case, we have to neglect the symbol $\chi$ ). If this ratio rushes to unity, the shown above consideration requires more careful approach.

We have to direct our attention to the limited precision of shown here final formulae. One from the reasons of that is related with traditional limited exactness for regularization of integrals over the transfer momentum at Coulomb scattering (see [16-18]).

\section{References}

1. Lifshits E.M. and Pitaevskiy L.P. Physical Kinetics. Nauka, Moscow, 1984 (in Russian).

2. Bogolubov N.N. Lections for Quantum Statistics. Radianska Shkola, 1949 (in Ukrainian).

3. Bogolubov N.N. Collected works in 12 volumes, v. 5: Non-equilibrium Statistical Mechanics. Nauka, Moscow, 2006 (in Russian).

4. Klimontovich Yu.L. Statistical Physics. Nauka, Moscow, 1978 (in Russian).

5. Boiko I.I. Kinetics of Electron Gas Interacting with Fluctuating Potential. Naukova dumka, Kyiv, 1993 (in Russian).

6. Anselm A.I. Introduction to Semiconductor Theory. Prentice Hall, 1982. 
7. Gantmaher V.F. and Levinson I.B. Scattering of Current Carriers in Metals and Semiconductors. Nauka, Moscow, 1984 (in Russian).

8. Bird R.B., Steward W.E., Lightfoot E.N. Transport Phenomena. John Willey \& Sons, 2007.

9. Zubarev D.N. Non-equilibrium Statistical Thermodynamics. Nauka, Moscow, 1971 (in Russian).

10. Landau L.D. and Lifshits E.M. Quantum Mechanics. Nauka, Moscow, 1963 (in Russian).

11. Boiko I.I. Dependence of the collision integral on electric field. Semiconductor Physics, Quantum Electronics \& Optoelectronics. 2015. 18, No 2. P. 138-143.

12. Boiko I.I. Influence of field dependent form of collision integral on kinetic coefficients. Semiconductor Physics, Quantum Electronics \& Optoelectronics. 2016. 19, No 2. P. 173-182.

13. Boiko I.I. Transport of Band Carriers in Semiconductor. Palmarium Academic Publishing, Saarbruken, 2013 (in Russian).

14. A.I. Achiezer (Ed.) Electrodynamics of Plasma. Nauka, Moscow, 1974 (in Russian).

15. Boiko I.I. Comparison of some different ways used for approximate solution of kinetic equation and calculations of carrier mobility. Semiconductor Physics, Quantum Electronics \& Optoelectronics. 20, No 4. P. 447-457.

16. Lennard A. On Bogolubovs kinetic equation for a spatially homogenous plasma. Ann. Phys. 1960. 3. P. 90.

17. Balescu R. Dynamical correlation patterns: A new representation of the Liouville equation. Physica. 1971. 56, No 1. P. 1-24.

18. Boiko I.I. Transport of Carriers in Semiconductors. Published by V. Lashkaryov Institute of Semiconductor Physics, NAS of Ukraine, Kyiv, 1993 (in Russian). 